\title{
Estrategias lúdicas dirigidas a la enseñanza de la matemática a nivel de Educación Primaria
}

\author{
Playful strategies for teaching mathematics at the nevel of Primary Education
}

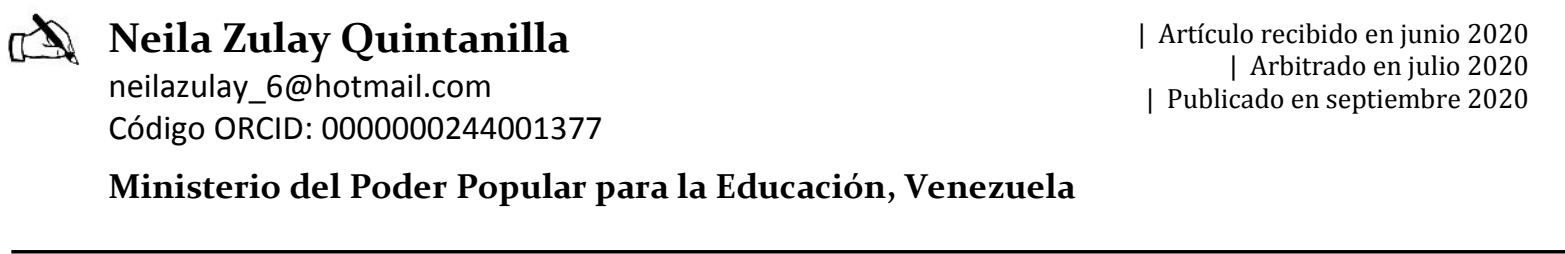

\section{Resumen}

La investigación tuvo como finalidad proponer estrategias lúdicas dirigidas a la enseñanza de la matemática a nivel del primer grado de educación primaria de la Escuela Básica Estadal "Profesora Teresa de Jesús Narza". Se enmarcó en un diseño no experimental de campo, nivel descriptivo bajo la modalidad de un proyecto factible. La muestra estuvo conformada por los seis (6) docentes del primer grado. La técnica aplicada fue la encuesta y el instrumento el cuestionario. Se concluyó que hay una carencia en la aplicación de estrategias lúdicas, que los docentes reconocen que la enseñanza de la matemática en los niños debe ser orientada de forma práctica y mediante el uso del juego, pero ellos no poseen las estrategias necesarias o desconocen cuál aplicar. Esta realidad sustentó la propuesta de conformar una serie de estrategias lúdicas, divertidas y pertinentes para brindar un aprendizaje significativo de la matemática.

\footnotetext{
Abstract

The purpose of the research was to propose playful strategies aimed at teaching mathematics at the level of the first grade of primary education at the State Basic School "Professor Teresa de Jesús Narza". It was framed in a non-experimental field design, descriptive level in the form of a feasible project. The sample was made up of the six (6) first grade teachers. The applied technique was the survey and the instrument was the questionnaire. Among the conclusions there is a lack in the application of playful strategies, where the teachers do recognize that the teaching of mathematics must be oriented in a practical way and through the use of the game, but they do not have the necessary strategies or do not know which one to apply. Reality that supports the proposal made up of a series of playful, fun and relevant strategies to provide meaningful learning of mathematics.
}

Palabras clave:

Estrategias lúdicas; enseñanza de la matemática; Educación primaria

\section{Keywords:}

Playful strategies; teaching mathematics; Primary education 
INTRODUCCIÓN

La educación es un componente esencial en la transformación de todo ser humano. Al educarse, una persona adquiere nuevos conocimientos, por lo tanto, la educación debe ser de calidad, eficiente y efectiva, que esté entrelazada con las nuevas generaciones, transformaciones, innovaciones y comunicaciones imperativas, que se identifique con el desarrollo socioeconómico y político del país. Esta idea concuerda con lo establecido en la Constitución de la República Bolivariana de Venezuela (1999) en su artículo 103: "Toda persona tiene derecho a una educación integral, de calidad, permanente, en igualdad de condiciones y oportunidades, sin más limitaciones que las derivadas de sus aptitudes, vocación y aspiraciones (...)" (p.93)

De esta manera, el modelo educativo debe estar en la capacidad de adaptarse a un mundo cambiante, complejo e incierto, donde constantemente aparecen avances en los sistemas de información y teorías que dan un nuevo enfoque al proceso educativo. Esto se evidencia en el ámbito laboral, caracterizado por novedosos métodos de producción que requieren un recurso humano altamente capacitado y tecnificado, capaz de a bordar el sistema de producción con una visión de conjunto, donde los procedimientos, estrategias innovadoras y creativas son una constante.

Desde este enfoque, la educación venezolana se encuentra en desventaja por diversos factores como lo son: la deserción escolar, la deficiencia en el aprendizaje, la falta de estrategias que propicien escenarios didácticos cónsonos con las necesidades de los estudiantes, el bajo rendimiento académico, la desmotivación, baja autoestima en algunos estudiantes, en fin, problemas de diversa índole. En concordancia con lo antes expuesto, Lugo (2013) citando a Vega señaló lo siguiente: "la deserción escolar en Venezuela, es el resultado de un sistema educativo que no está funcionando bien" (p.296).

En lo que respecta al desarrollo de la etapa de educación primaria en el subsistema de educación básica, el Ministerio del Poder Popular para la Educación estableció el Currículo Nacional Bolivariano (2007), el cual especifica los lineamientos a seguir para el desarrollo de los contenidos programáticos. Las autoridades institucionales y sobre todo los docentes, son los responsables de la implementación del mismo y de formar a un ciudadano apto para actuar con valores de justicia social y paz, siendo agente de cambio en el contexto que le toca vivir. Dentro de estas perspectivas, es indispensable que los niños y niñas se apropien del conocimiento matemático a lo largo de su desarrollo personal y profesional de modo que puedan aplicarlo en cualquier contexto. Al respecto, el Currículo Nacional Bolivariano (2007) afirma que:

Existen áreas del conocimiento tales como: el Área de Matemática, Ciencias Naturales y Sociedad, donde se concibe las matemáticas como una implicación de interconexión con las ciencias, cuya finalidad es ser un motor generador de cambios de transformaciones tendente a la liberación del ser humano, pues el dominar el lenguaje matemático influirá de manera significativa en la toma de decisiones, construcción e igual en la resolución de problemas en lo individual y en lo colectivo. (p.22) 
De allí, la importancia de la enseñanza de la matemática, la de colaborar en la capacitación de individuos integrales, con personalidad autocrítica frente a sus propias situaciones. Según el Currículo Nacional Bolivariano (2007):

En el logro de estos objetivos, la enseñanza de la matemática debe estar en concordancia con los contenidos programáticos y el nivel lingüístico de los niños y las niñas. "así, actividades como contar, medir, estimar, jugar, explicar y demostrar son importantes en el proceso de orientación y aprendizaje de las matemáticas, aunado al desarrollo de procesos como: representar, sintetizar, generalizar, abstraer, conjeturar y comunicar, entre otros. (p.21)

Por esta razón, la función social le asigna a la escuela entre uno de sus principios básicos, la responsabilidad de desarrollar las potencialidades matemáticas esenciales de los niños, niñas y adolescentes. Para ello, es importante disponer de un docente conocedor de estrategias que respondan de manera eficiente ante distintas problemáticas, ayudando al estudiante a generar diversas formas de pensamientos lógico y abstracto para responder eficazmente ante los numerosos procesos matemáticos presentes en el contexto que le rodea.

Respecto a esta idea, se puede destacar el estudio realizado por Pinto (2013) donde afirma que las necesidades de formación de los ciudadanos se prolongan más allá de la primera escolarización y se extienden a lo largo de toda la vida. En consecuencia, en el campo de la educación, es prioritaria la formación académica continua del docente en ejercicio para la asimilación y adaptación del aula ante los cambios que la sociedad impone.

Particularmente, en el campo de la matemática, la actualización permanente del docente de educación básica es imprescindible debido a las exigencias derivadas tanto del entorno social y personal, como profesional. Además sugiere que en el caso del área de matemática, es necesario superar el convencionalismo tradicional. El aporte de este antecedente permite asegurar que los educadores del área de matemáticas son requeridos en el desarrollo de competencias pedagógicas para enfrentar las tendencias actuales de esta era del conocimiento, teniendo así, la responsabilidad de capacitar al educando en la resolución de problemas relacionados con el entorno educativo, el contexto laboral y la vida cotidiana.

En este mismo orden de ideas, la presente investigación coincide con la problemática expuesta en la tesis de Rivero (2012) donde se explica que la enseñanza de las matemáticas, ha venido confrontado serios problemas debido a que se imparte de forma abstracta, la metodología utilizada no es la más adecuada, el aprendizaje de la misma se ha enfocado en la repetición de información memorística sin comprenderla y en la aplicación de metodologías mecánicas, lo que le complica al estudiante la resolución de problemas y por ende, dar resultados correctos al realizar ejercicios matemáticos.

Como respuesta a esta problemática, la investigación de Rivero (2012) se propuso analizar las estrategias didácticas para el desarrollo del pensamiento lógico-matemático en los estudiantes de Educación Primaria en la U.E. Arquidiocesana "Manolo Muchacho". Los resultados de dicha investigación determinaron que cuando el docente aplicó 
estrategias didácticas en la enseñanza de las matemáticas, el 44,1 por ciento de los estudiantes aumentó considerablemente el desarrollo lógico-matemático, razón por la cual se les recomendó participar en talleres psicoeducativos.

De este modo, el estudio de Rivero (2012) se relaciona con la presente investigación porque expone la necesidad en torno al manejo de estrategias didácticas adecuadas para la enseñanza de las matemáticas. Por lo tanto, sugiere a los docentes participar en diversos talleres, cursos, $\mathrm{u}$ otros encuentros pedagógicos que les permita apropiarse de métodos de enseñanza innovadores a fin de mejorar su labor educativa.

En otro orden de ideas, pero tomando en cuenta lo anteriormente expuesto, cabe destacar también en esta investigación el hecho de que todo conocimiento es construido. Por lo tanto, el docente debe asumir el rol de mediador y promotor de escenarios didácticos claves para propiciar un proceso de análisis reflexivo y de construcción. De este modo, el aprendiz está constantemente construyendo su propio conocimiento. Al respecto, Matos (2000) considera que:

El docente es un mediador no de manera declarativa, de hecho, debe asumir el reto de involucrarse en la construcción del conocimiento en el aula. Dentro de la praxis pedagógica integradora, el rol del docente debe ser percibido como promotor del aprendizaje, motivador y sensible. (p. 25)

Es por tanto que, el docente debe comprender que la matemática es fundamental en el desarrollo intelectual de los niños, que esta ciencia les ayuda a ser lógicos a partir de un proceso de razonamiento acorde a su crecimiento. Aunado a esto, es parte fundamental de su vida en sociedad. En este sentido, la escuela debe propiciar un ambiente armónico y de confianza para todos los participantes del hecho educativo, donde la enseñanza sea didáctica y dinámica. Es decir, un espacio donde el estudiante sea capaz de crear su propio conocimiento a través del aprendizaje significativo, que de acuerdo con Ausubel (1986) "ocurre cuando el sujeto consigue relacionar la nueva información con sus conocimientos previos" (p.34)

Esta correspondencia sucede cuando el sujeto tiene o muestra interés en el tema a tratar, de esta manera el aprendizaje será efectivo, duradero e interesante. En pocas palabras, el nuevo conocimiento se establece de una forma no arbitraria, pues el sujeto percibe la utilidad de lo que está aprendiendo. Es por esta razón que la enseñanza de la matemática debe estar basada en el aprendizaje significativo y que le permita al docente, mediante el empleo de estrategias didácticas, ser un mediador entre el nuevo conocimiento y los que ya posee el estudiante. Al respecto, Piaget, citado en Ramos (2013) establece en su teoría del funcionamiento de la inteligencia, dos procesos:

uno de asimilación, como el modo en que un organismo se encuentra a un estímulo del entorno en términos de organización actual, y el otro proceso de acomodación referido a la modificación de la organización actual como respuesta a la demanda del medio". (p.3)

Esto permitirá a los estudiantes adquirir los conocimientos de una manera simple y 
dinámica, pues los nuevos aprendizajes no serán tan complejos de lograr por una noción previa antes obtenida; es decir, la enseñanza de la matemática no debe ser de manera conductual con métodos pre-escritos en los textos, pues el docente como enlace entre el conocimiento y los aprendices y aprovechando sus capacidades como investigador, debe de indagar y experimentar cuáles son las estrategias, metodologías y herramientas para generar un aprendizaje efectivo de la matemática.

Sin embargo, vale la pena mencionar que, para cualquier buen docente, el hecho de transmitir un contenido a sus educandos es aparentemente sencillo, pero hay aspectos implicados que obstaculizan esta importante labor, como los tipos de aprendizajes, el contexto social y familiar de los educandos y todos aquellos factores que crean una compleja realidad e impiden el logro de los objetivos y competencias que se pautan en la planificación.

Como respuesta a lo expresado anteriormente, es necesario señalar las ventajas que tiene la aplicación de estrategias de aprendizaje significativo en los procesos de enseñanza y el rendimiento académico del educando. Por esta razón se puede decir que el docente es el primero que debe utilizar estrategias novedosas en la enseñanza de las matemáticas. De este modo, la metodología más apropiada para enseñar a los niños de educación primaria es el juego, definido por Flinchum (1988) (citado en Montero y Alvarado 2001) como aquel que "abastece al niño de libertad para liberar la energía que tiene reprimida," (p.20).

Por lo tanto, como el juego es considerado una estrategia pertinente a nivel de educación primaria, esta investigación se fundamentó en la construcción de estrategia de enseñanza de acuerdo a la perspectiva de Díaz-Barriga (2002), la cual señala:

Considerar las características generales de los aprendices (nivel de desarrollo cognitivo, conocimientos previos, factores motivacionales, entre otros): se describen las características generales para elaborar las estrategias de aprendizajes. Tipo de dominio del conocimiento en general y del contenido curricular en particular a abordar: hay que tener en claro cuál es el objetivo de aprendizaje y el contenido curricular requerido. Los objetivos a lograr, así como las actividades cognitivas y pedagógicas que debe realizar el estudiante con el propósito de conseguirlos: tener claro la meta a lograr, también las actividades cognitivas 0 pedagógicas necesarias para ello. Vigilancia constante del proceso de enseñanza (de las estrategias de enseñanza empleadas), así como del proceso y el aprendizaje de los estudiantes: vigilar constantemente los resultados a lo largo de la aplicabilidad de la estrategia. Determinar el contexto intersubjetivo (el conocimiento ya compartido), creado con los estudiantes: determinar el conocimiento ya impartido y creado por los estudiantes hasta ese momento del proceso de enseñanza. (p.9)

Por lo tanto, los juegos son actividades que despiertan el interés de los estudiantes de primaria y los mantiene concentrados en el logro de determinadas acciones y objetivos, además es un espacio que les 
permite descargar el exceso de energía, la cual pueden aprovechar en la creación nuevas estructuras del conocimiento. En este sentido, los participantes del hecho lúdico, pueden asumir el proceso educativo como algo divertido e innovador en su desarrollo. Por su parte, Vygotsky (1971, citado por Díaz-Barriga, 2002) afirma que:

El juego es un espacio de construcción de una semiótica y hace posible el desarrollo del pensamiento conceptual y teórico, considerando que el niño a partir de sus experiencias va formando conceptos, con un carácter descriptivo y referencial en cuanto se hallan circunscritos a las características físicas de los objetos. (p. 38)

Como resultado de la problemática planteada anteriormente y dadas las ventajas mencionadas acerca de las actividades lúdicas en el aprendizaje, surgen las siguientes interrogantes:

- ¿Qué estrategias utilizan los docentes en la enseñanza de la matemática en los niños y las niñas de primer grado?

- ¿Qué factibilidad tiene el uso del juego como estrategia de enseñanza en el área de matemática?

- ¿Cómo diseñar las estrategias lúdicas de enseñanza de la matemática?

En función a estas interrogantes, se estableció el siguiente objetivo general: proponer estrategias lúdicas dirigidas a la enseñanza de la matemática a nivel del primer grado de educación primaria de la Escuela Básica Estadal "Profesora Teresa de Jesús Narza” de la Parroquia Miguel Peña del Municipio Valencia del Estado Carabobo. Para el logro de este objetivo general, se plantean a continuación los siguientes objetivos específicos: diagnosticar la necesidad de estrategias dirigidas a la enseñanza de la matemática en los niños y las niñas de primer grado, en este sentido, se procedió a determinar la factibilidad de la propuesta, para así diseñar estrategias lúdicas dirigidas a la enseñanza de la matemática.

Es por tanto que, la presente investigación se enfocó en diseñar estrategias lúdicas dirigidas a la enseñanza de la matemática utilizadas por los docentes de la institución ya mencionada, implementando el juego como metodología principal para el logro de un aprendizaje significativo y el pleno desarrollo del educando. Cabe destacar la importancia de que los educandos se interesen por las matemáticas y sientan gusto por ella. Para lograr dicha motivación es pertinente proponer actividades contextualizadas de acuerdo a su edad e intereses.

Esta propuesta educativa se traduce en un extendido beneficio a los docentes, porque les brinda conocimientos nuevos respecto a estrategias lúdicas que son de gran apoyo al momento de abordar la asignatura de matemática en primer grado. Beneficia también a los educandos porque les proporciona un docente capacitado para enseñar efectivamente los contenidos programáticos del área. Además, se considera la relevancia pedagógica del estudio, por la razón de que considera el juego como una estrategia de enseñanza, representando una herramienta favorecedora de un proceso educativo donde se involucran estudiantes y docentes, promoviendo así, escenarios didáctico contextualizados y atractivos para los estudiantes. 


\section{MÉTODO}

La investigación desarrollada fue de carácter cuantitativo, asumió un diseño no experimental de campo, los datos fueron recolectados directamente del contexto abordado, en este caso el objeto de estudio comprende a los docentes de la Escuela Básica Estadal "Profesora Teresa de Jesús Narza".

De igual forma estuvo basada en una investigación descriptiva porque fue necesario describir, registrar, analizar e interpretar las actividades a desarrollar para fomentar la actuación del personal ante una comunicación institucional y describir las estrategias de comunicación efectivas ya existentes en la institución. Asimismo, se enmarcó en la modalidad de proyecto factible, que según la Universidad Pedagógica Experimental Libertador (UPEL) (2014) se define como "la elaboración y desarrollo de una propuesta de un modelo operativo viable dirigido a solucionar problemas, o necesidades de organizaciones o grupos sociales; puede referirse a la formulación de políticas, programas, tecnologías, métodos o procesos" (p. 2l)

En continuidad con la idea anterior, el presente estudio se desarrolló en atención a tres fases en correspondencia con los objetivos específicos establecidos: en primer lugar, la fase diagnóstica: la cual según Orozco, Labrador y Palencia (2002) se define como "una reconstrucción del objeto de estudios y tienen por finalidad detectar situaciones donde se ponga de manifiesto la necesidad de realizarlo" (p.186). Al culminar esta fase se pudo obtener la información necesaria para la propuesta y se continuó con el siguiente objetivo: propiciar cambios en la enseñanza de la matemática a través de la creación de experiencias o escenarios didácticos significativos para los estudiantes de educación primaria.

Seguidamente, se realizó el estudio de la factibilidad, la cual Gómez (2000), define como "la posibilidad de desarrollar un proyecto, tomando en consideración la necesidad detectada, beneficios, recursos humanos, técnicos, financieros, estudios de mercado..." (p.26). Cabe destacar también, que se analizó el carácter técnico y económico, de lo que se concluyó que esta investigación no implica gastos elevados para implementarla y en la parte institucional, está apegada al currículo respectivo. Por lo tanto, se puede decir que la misma es factible. Por ello, una vez culminado el diagnóstico y determinada la factibilidad, se procedió a la elaboración de la propuesta, lo que generó necesariamente una tercera fase de la investigación.

Con respecto a la población y objeto de estudio, estuvo conformada por seis (06) docentes de primer grado de la Escuela Básica Estadal "Profesora Teresa de Jesús Narza", ubicada en la Parroquia Miguel Peña, Municipio Valencia, estado Carabobo. Se tomó el cien por ciento de la población, debido a que fue de fácil acceso al investigador, por lo tanto, se constituyó en un estudio censal, es decir, sin muestra de estudio, sino que abarca a toda la población. Al respecto Arias (2006) afirma que, "si la población, por el número de unidades integradoras, resulta accesible en su totalidad, no será necesario extraer una muestra. En consecuencia, se podrá investigar u obtener datos de toda la población" (p. 83)

En cuanto a la selección de técnicas e instrumentos de recolección de información se utilizó la técnica de la encuesta, que según Palella y Martins (2010) está: 
"destinada a obtener datos de varias personas cuyas opiniones interesan al investigador" (p. 123). A través de dicha encuesta se pudo conocer las opiniones, las actitudes y los comportamientos de varias personas en correspondencia a un contenido en específico. Aunado a esto, como instrumento de recolección de datos se aplicó un cuestionario de preguntas cerradas. El mismo estuvo estructurado por preguntas dicotómicas solo con dos opciones de respuestas ( $\mathrm{Si}$ y $\mathrm{No}$ ). Fue validado por el juicio de diversos expertos y la confiabilidad obtenida fue de $\mathrm{Kr}=0,96$ según la fórmula de Kuder Richardson por lo cual el instrumento fue definido como confiable, lo que permitió continuar con la ruta metodológica.
RESULTADOS Y DISCUSIÓN

Una vez realizada la recolección de los datos, se construyeron tablas de distribución de frecuencias y gráficos por cada ítem con los resultados obtenidos. A continuación, se describen los cuatro ítems más relevantes.

Para comenzar, en el ítem número 2 donde se pregunta al encuestado si iLa enseñanza de la matemática es importante en el aprendizaje de las áreas académicas complementarias en la formación integral del niño y la niña? se demostró que de un total de 6 docentes encuestados, los cuales representan el 100 por ciento, afirmaron lo importante de la enseñanza de la matemática como área complementaria. Esto se puede apreciar en el siguiente gráfico.

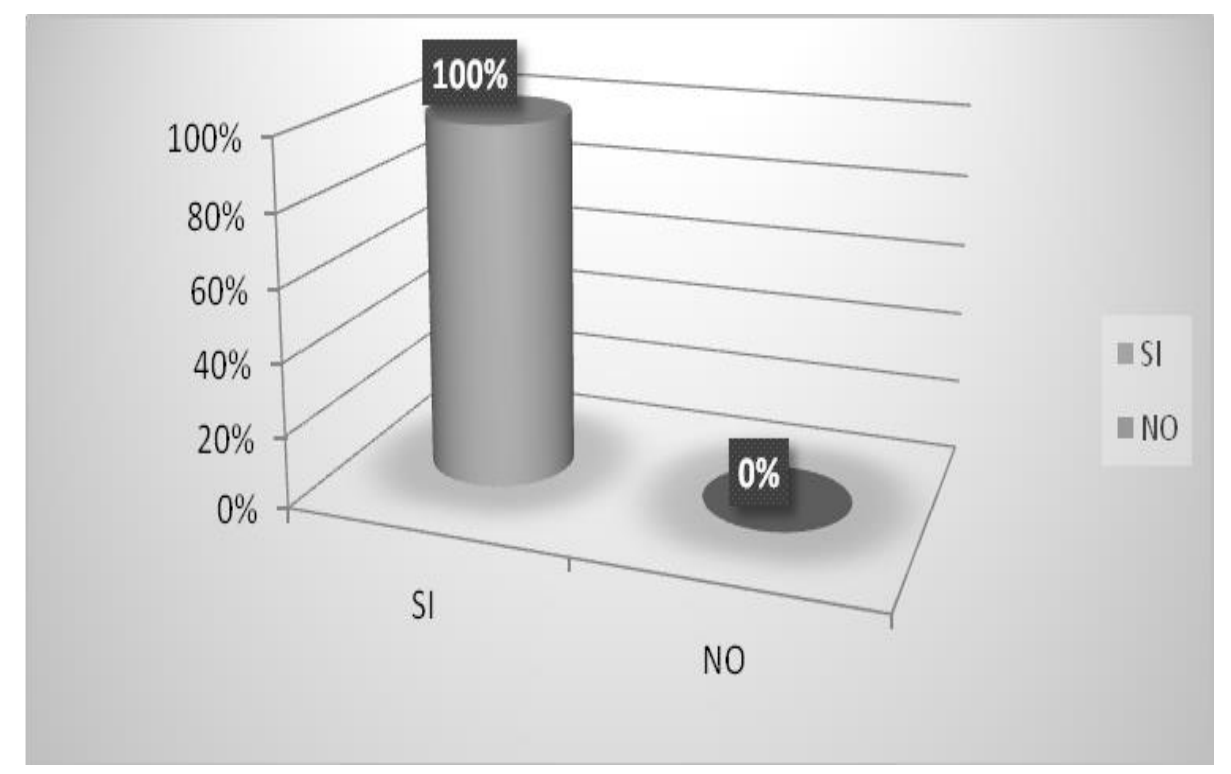

Gráfico 1. Importancia de la enseñanza de la matemática. (Fuente: Quintanilla, 2020) 
En tal sentido, Vygotsky (citado por Díaz-Barriga, 2002) considera que "el conocimiento no es un objeto que se pasa de uno a otro, sino se construye por medio de operaciones y habilidades cognoscitivas que se inducen en la interacción social" (p.12). Visto de esta forma, en el campo de la enseñanza de las áreas académicas complementarias, se desarrollará siempre el contenido comenzando de lo más simple hasta lo más complejo, teniendo como particularidad que el conocimiento adquirido, una vez procesado no se olvida, porque la experiencia no proviene de los objetivos sino de su acción sobre los mismos.
Seguidamente, en el Ítem 4 se les preguntó a los docentes: iIncluye usted alguna estrategia de enseñanza en su planificación diaria para el aprendizaje de la matemática? Como resultado se evidencia que, de un total de 6 docentes encuestados, el 17 por ciento de los mismos correspondientes a un solo de ellos, afirma incluir algunas estrategias de enseñanza en su planificación diaria mientras que los otros 5, es decir, el 83 por ciento restante no aplican lo antes mencionado. Esto se puede apreciar en el siguiente gráfico.

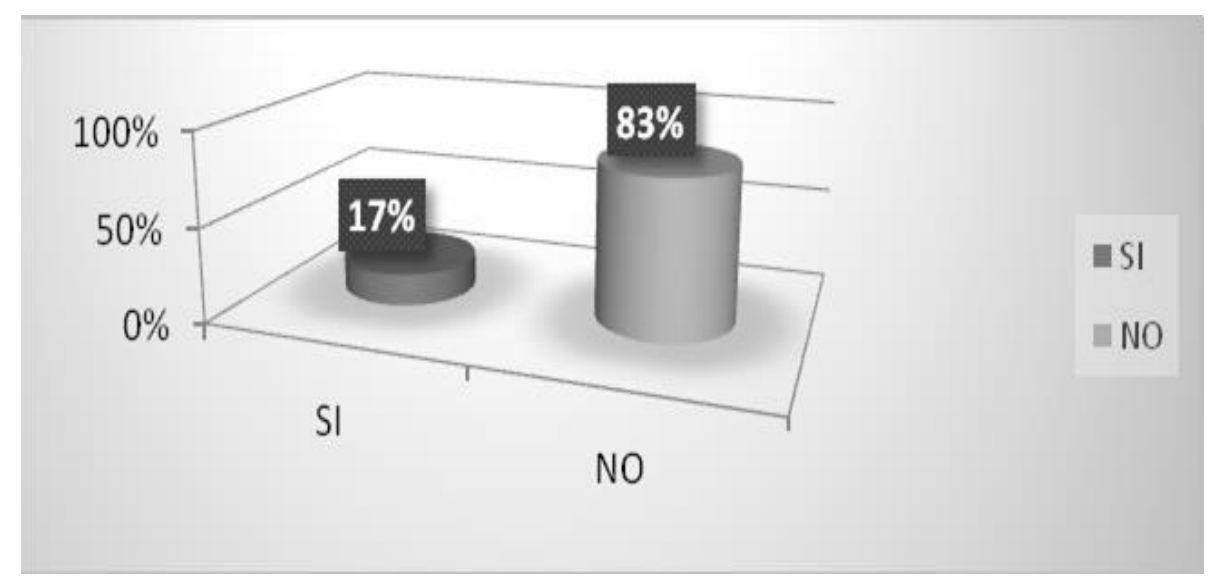

Gráfico 2. Inclusión de estrategia de enseñanza. (Fuente: Quintanilla, 2020)

En función a los resultados obtenidos en torno a la inclusión de estrategias de enseñanza, al respecto Díaz-Barriga (2002) señala que: "las estrategias seleccionadas por el docente, deben garantizar una alta efectividad, al ser utilizadas como apoyo en la dinámica del proceso de enseñanza y aprendizaje, deben incluirse al inicio, durante y al final de una sesión pedagógica" (p. 69).

En concordancia con esta última idea, y tomando en cuenta el resultado arrojado en el ítem 4, es necesario considerar la relevancia que tiene al momento de dar clases, el tener una buena planificación con la que el docente se pueda guiar, enfocar y apoyar en el cumplimiento de las fases de una sesión pedagógica, teniendo sus respectivas estrategias ya definidas y preparadas con antelación. El hecho de planificar es pues, la organización del trabajo diario posterior a un diagnóstico de habilidades y conocimientos previos que se hace al estudiante para detectar fortalezas o deficiencias en sus capacidades y así enfocar la clase de una forma más certera. Esto facilita el logro de las competencias y objeticos previamente establecidos. 
Otro Ítem relevante en esta no motivan a sus estudiantes a través de investigación fue el número 8: ¿Motiva a sus educandas al logro de los contenidos por medio de estrategias lúdicas? En este caso el estrategias lúdicas. Se puede apreciar de manera más clara en el Gráfico 3 presentado $87 \%$ de los entrevistados respondieron que a continuación.

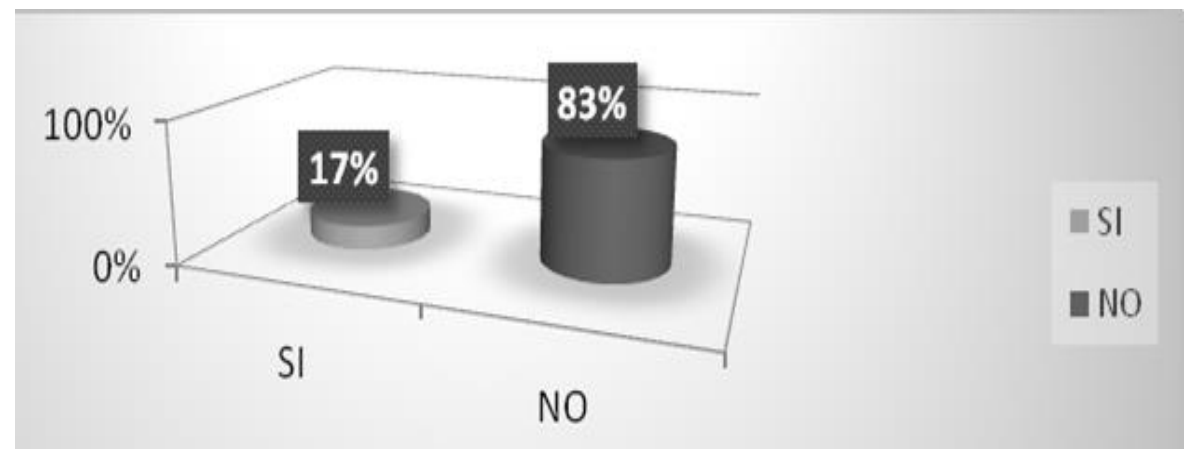

Gráfico 3. Logro de los contenidos por medio de estrategia lúdicas. (Fuente: Quintanilla, 2020)

En este sentido, Piaget (1986) afirma que: "El juego forma parte de la inteligencia del niño, representa la asimilación funcional o reproductiva de la realidad según cada etapa evolutiva del individuo" (p. 115). Eso significa que las capacidades sensorio-motrices, simbólicas o de razonamiento, son consideradas como aspectos esenciales en el desarrollo del individuo, ya que condicionan el origen y la evolución del juego.

Otra pregunta relevante es la correspondiente al ítem 10: ¿Considera usted que al utilizar el juego como una estrategia de enseñanza, los educandos obtengan las habilidades necesarias para adquirir los conocimientos previstos? A través de los resultados del ítem, representados en el Gráfico 4, se evidencia que de un total de 6 docentes encuestados los cuales representan el 100 por ciento de los mismos, afirman que en los educandos se pueden desarrollar habilidades necesarias utilizando el juego como una estrategia de enseñanza en el aula.

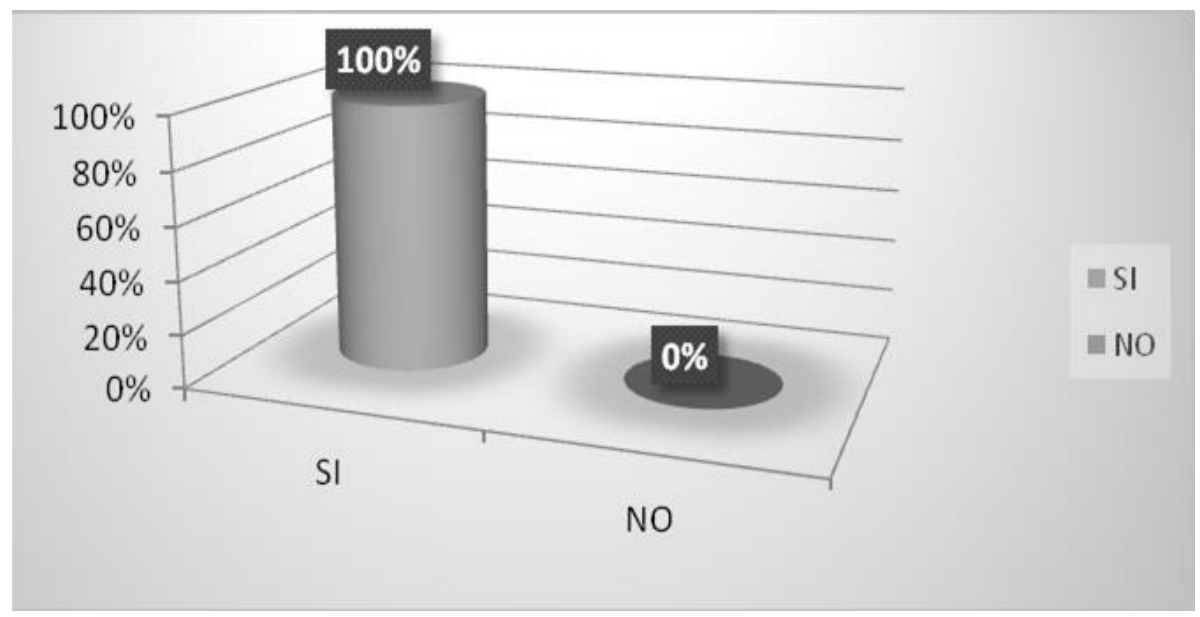

Gráfico 4. Habilidades necesarias para adquirir conocimientos previstos. (Fuente: Quintanilla, 2020) 
En concordancia con lo anterior, Yturralde (2009) señala que:

Los juegos lúdicos pueden estar presentes en las diferentes etapas de los procesos de aprendizaje del ser humano. Es evidente el valor educativo, que el juego lúdico tiene y en la escuela en general, fomenta el desarrollo psicosocial, la conformación de la personalidad, evidencia valores, puede orientarse a la adquisición de saberes, encerrando una amplia gama de actividades interactúan el placer, el gozo, la creatividad y el conocimiento. (p. 85)

De este modo, dada la versatilidad y la amplia variedad de posibilidades de aprendizaje que brinda el hecho de jugar, convierte esta actividad en una oportunidad valiosa al momento de abordar los contenidos matemáticos ya que despierta motivación, interés y gusto en el estudiante que participa de este tipo de actividades obteniendo así, experiencias significativas, conocimiento, desarrollo de destrezas y habilidades, entre otros aspectos.

\section{CONCLUSIONES}

Una vez efectuado el diagnóstico y el análisis de los datos sobre la base de los objetivos propuestos orientadores de la investigación, así como el análisis de las repuestas dadas por las unidades seleccionadas a través del cuestionario aplicado, se pudo evidenciar y concluir lo siguiente:

Los docentes que conforma la población de estudio en la presente investigación, emplean diversidad de técnicas y estrategias pedagógicas basadas en la práctica repetitiva de actividades poco significativas, como la copia o transcripción a mano, la repetición, la lectura no comprensiva de los libros de texto, entre otras. Se dedujo de este estudio que, el personal docente de la institución, no desarrolla estrategias lúdicas en sus clases que propicien el desarrollo creativo, la originalidad e innovación en la enseñanza de la matemática. De este modo, se limita al estudiante en el logro de un aprendizaje significativo, que induzca la valoración de la matemática y su fácil aplicabilidad en diversas áreas del conocimiento.

Aunado a esto, los juegos conforman una serie de estrategias lúdicas que tienen como objetivo, la adquisición de conocimientos y el aprendizaje mediante actividades desinteresadas, donde el estudiante adquiere diversos factores de motivación y aprende con una mínima dificultad. El rol del docente en este caso como orientador y mediador, consiste en la planificación y ejecución de una serie de técnicas y estrategias pedagógicas que faciliten y motiven al participante a aprender de forma divertida y armónica. También conviene la elaboración y uso de recursos didácticos que brinden la oportunidad a los estudiantes de manifestar los resultados obtenidos en el proceso cognitivo. En pocas palabras, el empleo de dichas estrategias y recursos favorece el proceso de enseñanza aprendizaje promoviendo un mayor rendimiento académico en los educandos.

Cabe destacar también el efecto positivo que se pudo observar en los docentes, durante y después de la experiencia en la implementación los juegos como estrategia para la enseñanza de la matemática. Según algunos de ellos, fue una experiencia satisfactoria. Puede describirse dicha experiencia como un momento de encuentro entre el conocimiento matemático y pedagogía, donde el docente puede atreverse a innovar, a proponer procesos nuevos, más 
dinámicos y abiertos a distintas posibilidades. De este modo, el docente cumple con proporcionar herramientas didácticas, permitiendo a los estudiantes alcanzar los objetivos planteados en la modalidad.

Es importante señalar que las actividades planificadas en el aula de clase merecen ser alcanzadas de forma placentera y armónica, ya que son un fenómeno complejo y multifactorial con elementos externos al estudiante como lo son el ambiente familiar, escolar y los docentes; o de elementos internos como los factores físicos, cognoscitivos, afectivos, de personalidad, de voluntad, de motivación, entre otros.

De igual forma, el docente es un factor que contribuye al crecimiento y desarrollo integral de sus estudiantes, y una de sus funciones es mediar y asistir en el proceso enseñanza y aprendizaje. Allí los estudiantes desarrollan sus conocimientos, capacidades, destrezas y actitudes en ambientes recreativos, ejercitan sus potencialidades y además, participan en actividades lúdicas. Por esta razón, se recomienda al docente dominar el saber pedagógico que consiste en el conocer, hacer y actuar.

En relación al último objetivo de investigación, se evidenció una estrecha relación entre el juego como estrategia lúdica y la enseñanza de la matemática. Es por ello que el diseño de estrategias lúdicas dirigidas a la enseñanza de las matemáticas propicia en el estudiante, el disfrute de las actividades, principalmente porque el juego, en cualquiera de sus manifestaciones pedagógicas es una estrategia que permite mantener la atención, el gusto, la motivación y el interés en los estudiantes, logrando una participación activa y una retroalimentación constante, favoreciendo aspectos como la convivencia social, el mejoramiento en la resolución de operaciones matemáticas, superación de la apatía producida por el método de enseñanza tradicional, el gusto por la permanencia en la institución, desarrollo integral y crecimiento personal.

Dadas las conclusiones anteriores, se recomienda el uso de estrategias de enseñanza basadas en el juego que faciliten la comprensión en cuanto a la solución de operaciones matemáticas en los primeros años de educación primaria. Esta recomendación se hace porque el juego como estrategia tiene un carácter innovador, motivante, promociona el amor por la matemática y es una forma para producir aprendizajes de manera natural, creativa, auténtica y espontánea por parte de los aprendices.

Un ejemplo de estrategia lúdica para la enseñanza de la matemática es la propuesta "Jugando me divierto y aprendo Matemática", la cual responde a la necesidad de proponer el juego como estrategia lúdica en los estudiantes pertenecientes a la Escuela Básica Estadal "Profesora Teresa de Jesús Narza". A continuación, se presentan tres estrategias lúdicas que forman parte de dicha propuesta, de un total de 14 realizadas. Las mismas están estructuradas con inicio, desarrollo y cierre.

Es importante destacar que en esta propuesta se emplearon los postulados de Díaz-Barriga (2002), las cuales se diseñaron tomando en cuenta el contenido programático del área de aprendizaje de "Matemática, Ciencias Naturales y Sociedad", específicamente la noción de números basándose principalmente en el juego. En este sentido, se consideraron las características generales de los aprendices, el dominio del conocimiento en general y del contenido curricular por parte del docente de primer grado, estableciéndose tanto los objetivos como metas que se desean lograr en conjunto con las 
actividades cognitivas y pedagógicas. Todo esto, monitoreando el proceso de enseñanza que aplican los docentes para poder determinar el contexto intersubjetivo, es decir, entre los que enseñan y aprenden dejando abierta la posibilidad de que el proceso puede ir mejorando y evolucionando.

La primera de las estrategias a desarrollar se titula: iEl que llegue primero! cuyo contenido está enfocado en construcción del concepto básico de la adición. Se centra en crear la noción de cantidad numérica a través del juego. Para realizar esta actividad, cada niño debe tener un tablero y cincuenta objetos pequeños (frijoles, piedritas, etc.) Los participantes deben estar agrupados en equipos de cinco, y para cada equipo de cinco debe haber dos dados.

Posteriormente para desarrollar la actividad, cada jugador tira dos dados por turno, suma los puntos que obtuvo y coloca en cada una de las casillas del tablero un objeto hasta completar el total de puntos obtenidos. Finalmente, en el cierre, gana el primero que llene el tablero. Como sugerencia, conforme el niño avanza puede aumentar el número de casillas y de dados para incrementar el rango numérico en el conteo.
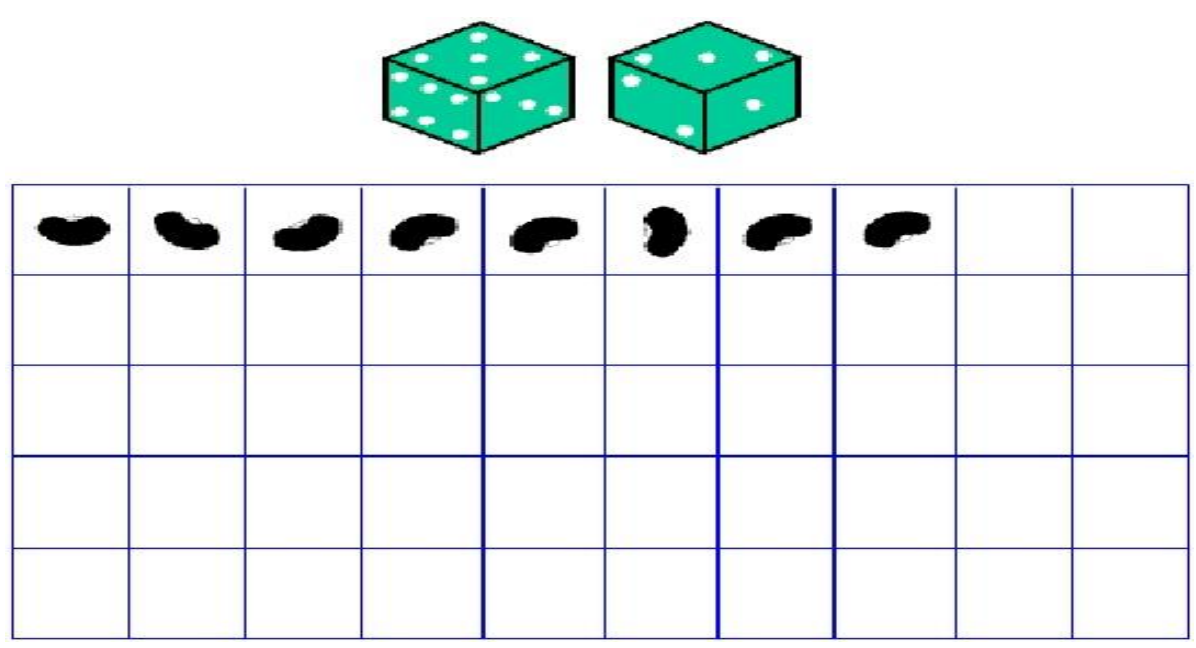

Figura l. Tablero alusivo a la estrategia iEl que llegue primero!

Otra estrategia es la denominada "Muñecos hambrientos" cuyo contenido está centrado en la clasificación de datos y el objetivo es construir el concepto de probabilidad. Para ello se necesitan los siguientes materiales: pelotas de colores, música y los muñecos que serán cajas. Para comenzar, se colocarán dos cajas (que serán los muñecos), a una distancia prudente y una fila de niños en correspondencia a cada caja. Seguidamente, para el desarrollo, los primeros niños de cada fila, lanzarán la bolita, insertándola dentro de la boca del muñeco en un tiempo determinado. Para el cierre de la ronda, cada uno deberá contar y sumar cuántos aciertos obtuvo. Luego se les pide a los participantes comparar quien logró mayor, menor o igual cantidad de aciertos. Después con cada muñeco se determinará cuál fue el color que más acertaron y por qué. Y con ayuda de las pelotas o bolitas se trabajará el concepto de probabilidad. 
La tercera estrategia lúdica se denomina "Jugando cartas". Está basada en el contenido de relación mayor que y menor que, y su objetivo educativo es comparar cantidades numéricas según las relaciones mayor que y menor que. Los recursos que se necesitan para aplicar esta estrategia son cartas con dibujos del 1 al 10. Para iniciar el juego, el docente debe dividir al grupo en parejas, tríos o cuartetos de jugadores dependiendo de la cantidad de participantes que estén. A cada grupo se le debe repartir un juego de diez cartas con ilustraciones y números del 1 al 10. Para el desarrollo, cada grupo debe colocar su montón de diez cartas delante de sí, boca abajo. Los jugadores vuelven la carta de arriba al mismo tiempo y el que tenga la más alta se lleva todas las cartas que están boca arriba sobre la mesa. Si entre dos de los jugadores se produce un empate dirán "guerra" y cada uno pondrá junto a su carta, una carta boca abajo y otra vuelta hacia arriba. El que tenga la carta más alta se llevará todas las cartas de la partida en cuestión. Gana el grupo que tenga más cartas al finalizar.

Según las estrategias implementadas por los estudiantes y las que se pretendan promover, el docente decidirá si utilizar o no las cartas con dibujos. Al cierre de la actividad, para comparar números, los estudiantes podrán recurrir a diversas estrategias como, por ejemplo: establecer la correspondencia 1 a 1 entre las dos colecciones, establecer la correspondencia entre la colección y la serie numérica y comparar números. Si el objetivo del docente fuera que los estudiantes comparen los números apoyándose en la serie numérica (soporte lineal) será conveniente elegir las cartas del 1 al 10 sin dibujos. A partir de este material es probable que los estudiantes busquen los números obtenidos en sus cartas en una serie numérica y observen cuál es la relación entre ellos que permitirá indicar cuál es el ganador (el que está más lejos del comienzo).

A modo de cierre, se recomienda a los docentes que, al momento de planificar sus clases, tomen en cuenta las actividades lúdicas alusivas al aprendizaje de la matemática debido al impacto tan positivo que estas tienen en el aprendizaje. Además, dichas estrategias permiten un intercambio de saberes en ambientes de recreación, donde el niño y la niña participan y aportan sus ideas. Se considera esta recomendación con el fin de evitar el uso excesivo de la metodología tradicional, ya que el proceso de enseñanza y aprendizaje es responsabilidad también del docente cuya misión es enseñar a aprender, dinamizar y facilitar el aprendizaje de forma significativa.

\section{REFERENCIAS}

Arias, F. (2006). El proyecto de investigación: Guía para su elaboración. Tercera edición. Caracas, Venezuela: Episteme

Ausubel, D. (1986). Psicología educativa. 3era edición. México: Editorial Trillas

Constitución de la República Bolivariana de Venezuela (1999). Caracas, Venezuela. Gaceta Oficial 36.860, 30/12/1999. Caracas, Venezuela

Díaz-Barriga, F. (2002). Estrategias docentes para un aprendizaje significativo: una interpretación constructivista. D.F. México: McGraw-Hill Interamericana Editores, S. A. de C. V

Gómez, C. (2000). Proyectos factibles. Valencia, Venezuela: Predios

Lugo, B. (2013). La deserción estudiantil: irealmente es un problema social? Revista de Postgrado ARJÉ, 7(12), 289-309. Recuperado de: http://servicio.bc.uc. edu.ve/educacion/arje/arj12/artl7.pdf 
Matos, M (2000). Resiliencia. [Documento en línea] Recuperado de http://clubensayos.com/Ciencia/Resilien cia/30606.html

Ministerio Educación (2007). Currículo Nacional Bolivariano. Caracas, Venezuela: Fundación Centro Nacional para el Mejoramiento de la Enseñanza de Ciencia (CENAMEC)

Montero, M. M., y Alvarado, M. D. L. Á. M. (2001). El juego en los niños: un enfoque teórico. Revista educación, 25(2), 113-124

Orozco, C., Labrador, M., Palencia, A. (2002). Metodología. Venezuela: Editorial Clemente

Palella, S. y Martins, F. (2010). Metodología de la investigación cuantitativa. ( $2^{\mathrm{a}}$ ed. $)$ Caracas, Venezuela: Fondo Editorial de la Universidad Pedagógica Experimental Libertador

Piaget, J. (1980). La formación del símbolo en el niño. Fondo de la Cultura Económica (Edición original de 1959). D.F, México

Pinto, R. (2013). Alcances preliminares del proyecto Canaima respecto a la formación de competencias matemáticas en educación básica desde la perspectiva de sus actores (Tesis de maestría) Facultad de Ciencias de la Educación, Universidad de Carabobo. Valencia, Venezuela Recuperado de http://riuc.bc.uc.edu.ve/bitstream/123456789/13 21/1/rPinto.pdf

Ramos, G. (2013). Asimilación y acomodación de Piaget. Recuperado de: http://asimilacionyacomodacion.blogspo t.com/2013/02/la-asimilacion-yacomodacion-de-piaget.html

Rivero, Y. (2012). La planificación de estrategias didácticas y el desarrollo del pensamiento lógico-matemático en educación primaria. División de estudios para graduados. Universidad del Zulia (Venezuela)

Universidad Pedagógica Experimental Libertador (2014). Manual de Trabajo de Grado de Especialización, Maestría y Tesis Doctorales Caracas, Venezuela: F.E.D.U.P.E.L

Yturralde, E. (2009). La lúdica y el aprendizaje. D.F. México: Zenit 